\title{
Initial Conflicts for Transformation Rules with Nested Application Conditions
}

\author{
Leen Lambers ${ }^{1(\otimes)}\left(\mathbb{D}\right.$ and Fernando Orejas $^{2}$ (D) \\ 1 Hasso Plattner Institute, University of Potsdam, Potsdam, Germany \\ leen. lambers@hpi.de \\ 2 Universitat Politècnica de Catalunya, Barcelona, Spain \\ orejas@lsi.upc.edu
}

\begin{abstract}
We extend the theory of initial conflicts in the framework of $\mathcal{M}$-adhesive categories to transformation rules with ACs. We first show that for rules with ACs, conflicts are in general neither inherited from a bigger context any more, nor is it possible to find a finite and complete subset of finite conflicts as illustrated for the category of graphs. We define initial conflicts to be special so-called symbolic transformation pairs, and show that they are minimally complete (and in the case of graphs also finite) in this symbolic way. We show that initial conflicts represent a proper subset of critical pairs again. We moreover demonstrate that (analogous to the case of rules without ACs) for each conflict a unique initial conflict exists representing it. We conclude with presenting a sufficient condition illustrating important special cases for rules with ACs, where we do not only have initial conflicts being complete in a symbolic way, but also find complete (and in the case of graphs also finite) subsets of conflicts in the classical sense.
\end{abstract}

Keywords: Graph transformation $\cdot$ Critical pairs $\cdot$ Conflicts

\section{Introduction}

Detecting and analyzing conflicts is an important issue in software analysis and design, which has been addressed successfully using powerful techniques from graph transformation (see, e.g., $[12,15,17,24]$ ), most of them based on critical pair analysis. The power of critical pairs is a consequence of the fact that: a) they are complete, in the sense that they represent all conflicts; b) there is a finite number of them; and c) they can be computed statically. The main problem is that their computation has exponential complexity in the size of the preconditions of the rules. For this reason, some significantly smaller subsets of critical pairs that are still complete have been defined $[1,19,21]$, clearing the way for a more efficient computation. In particular, recently, in [19], a new approach

F. Orejas has been supported by the Salvador de Madariaga grant PRX18/00308 and by funds from the Spanish Research Agency (AEI) and the European Union (FEDER funds) under grant GRAMM (ref. TIN2017-86727-C2-1-R).

(C) Springer Nature Switzerland AG 2020

F. Gadducci and T. Kehrer (Eds.): ICGT 2020, LNCS 12150, pp. 109-127, 2020.

https://doi.org/10.1007/978-3-030-51372-6_7 
Table 1. Critical pairs versus initial conflicts

\begin{tabular}{l|l|l|l}
\hline & Plain rules & Rules with NACs & Rules with ACs \\
\hline $\begin{array}{l}\text { Critical pairs } \\
(\mathrm{CPs})\end{array}$ & $\begin{array}{l}\text { Subset of conflicts, } \\
\text { complete }[27]\end{array}$ & $\begin{array}{l}\text { Subset of conflicts, } \\
\text { complete }[17,20]\end{array}$ & $\begin{array}{l}\text { Symbolic, } \\
\text { complete }[6,9]\end{array}$ \\
\hline $\begin{array}{l}\text { Initial } \\
\text { conflicts }\end{array}$ & $\begin{array}{l}\text { Subset of conflicts, } \\
\text { min. complete, } \\
\text { proper subset of } \\
\text { CPs }[1,19]\end{array}$ & $\begin{array}{l}\text { Symbolic (Definition 10), } \\
\text { min. complete, regular } \\
\text { (Theorem 6) \& } \\
\text { conservative extension of } \\
\text { CPs (Theorem 7) }\end{array}$ & $\begin{array}{l}\text { Symbolic } \\
\text { (Definition 10), } \\
\text { min. complete, } \\
\text { proper subset of } \\
\text { CPs (Theorem 3) }\end{array}$ \\
\hline
\end{tabular}

for conflict detection was introduced based on a different intuition. Instead of considering conflicts in a minimal context, as for critical pairs, we used the notion of initiality to characterize a complete set of minimal conflicts, showing that initial conflicts form a strict subset of critical pairs. In particular, we have that every conflict is represented by a unique initial conflict, as opposed to the fact that each conflict may be represented by many critical pairs.

Most of the work on critical pairs only applies to plain graph transformation systems, i.e. transformation systems with unconditional rules. Nevertheless, in practice, we often need to limit the application of rules, defining some kind of application conditions (ACs). In this sense, in [17,20] we defined critical pairs for rules with negative application conditions (NACs), and in [6,9] for the general case of ACs, where conditions are as expressive as arbitrary first-order formulas on graphs. However, to our knowledge, no work has addressed up to now the problem of finding significantly smaller subsets of critical pairs for this kind of rules. In this paper we generalize the theory of initial conflicts to rules with ACs in the framework of $\mathcal{M}$-adhesive transformation systems. In particular, the main contributions of this paper (as summarized in Table 1) are:

- The definition of the notion of initial conflict for rules with ACs, based on a notion of symbolic transformation pair, showing that the set of initial conflicts is a proper subset of the set of critical pairs and that it is minimally complete ${ }^{1}$, in the sense that, no smaller set of symbolic transformation pairs exists that is also complete. In particular, the cardinality of the set of initial conflicts is, at most, the cardinality of the set of initial conflicts for the plain case, when disregarding the ACs, plus one. Moreover, as in the plain case, every conflict is an instance of a unique initial conflict.

- The identification of a class of regular initial conflicts that demonstrate a certain kind of regularity in their application conditions. This allows us to unfold them into a complete (and in the case of graphs also finite) subset of conflicts. In particular, we show that, in the case of rules with NACs, initial conflicts are regular, implying that our initial conflicts represent a conservative extension of the critical pair theory for rules with NACs.

The paper is organized as follows. We describe related work in Sect. 2 and, in Sect. 3, we present some preliminary material, where we also include some new

\footnotetext{
${ }^{1}$ Provided that the considered category has initial conflicts for the plain case.
} 
results. More precisely, in Subsect. 3.1 and Subsect. 3.2 we briefly reintroduce the framework of $\mathcal{M}$-adhesive categories and of rules with ACs; in Subsect.3.3 we reintroduce critical pairs for rules with ACs following [6,9]; in Subsect.3.4 we reintroduce initial conflicts for plain rules, and in Sect. 4 we introduce initial parallel independent transformation pairs. This result is used in Sect.4, where we present the main results of the paper about initial conflicts for rules with ACs. Then, in Sect. 5 we show our results on unfolding initial conflicts. Finally, we conclude in Sect. 6 discussing some future work. Detailed proofs can be found in the full version of the paper [23].

\section{Related Work}

Most work on checking confluence for rule-based rewriting systems is based on the seminal paper from Knuth and Bendix [14], who reduced the problem of checking local confluence to checking the joinability of a finite set of critical pairs. This technique has been extensively studied in the area of term rewriting systems (see, e.g., [25]), and it was introduced in the area of graph transformation by Plump [27] in the context of term-graph and hypergraph rewriting. Moreover, he also proved that (local) confluence of graph transformation systems is undecidable, even for terminating systems, as opposed to what happens in the area of term rewriting systems. However, recently, in [2] it is shown that confluence of terminating DPO transformation of graphs with interfaces is decidable. The authors explain that the reason is that interfaces play the same role as variables in term rewriting systems, where confluence is undecidable for terminating ground (i.e., without variables) systems, but decidable for non-ground ones.

Computing critical pairs in graph transformation, as introduced by Plump [27], is exponential in the size of the preconditions of the rules. For this reason, different proper subsets of critical pairs with a considerably reduced size were studied that are still complete $[1,19,21]$, clearing the way for a more efficient computation. The notion of essential critical pair [21] for graph transformation systems already allowed for a significant reduction, and, the notion of initial conflict [19], introduced for the more general $\mathcal{M}$-adhesive systems, allowed for an even larger reduction. However, not all $\mathcal{M}$-adhesive categories have initial conflicts. In [19] it is shown that typed graphs do have them and [1] extended that result proving that arbitrary $\mathcal{M}$-adhesive categories satisfying some given conditions also have initial conflicts.

A recent line of work concentrates on the development of multi-granular conflict detection techniques [3,18,24]. An extensive literature survey shows [24] that conflict detection is used at different levels of granularity depending on its application field. The overview shows that conflict detection can be used for the analysis and design phase of software systems (e.g. for finding inconsistencies in requirement specifications), for model-driven engineering (e.g. supporting model version management), for testing (e.g. generation of interesting test cases), or for optimizing rule-based computations (e.g. avoiding backtracking). These multigranular techniques are presented for rules without application conditions (ACs). 
Our work builds further foundations for providing multi-granular techniques also in the case of rules with ACs in the future.

The use of (negative) application conditions, to limit the application of graph transformation rules, was introduced in $[8,10]$, while the more general approach, using nested conditions, was introduced by Habel and Penneman [11]. Checking confluence for graph transformation systems with application conditions (ACs) has been studied in $[17,20]$ for the case of negative application conditions (NACs), and in [6,9] for the more general case of ACs. However, it is an open issue to find proper subsets of critical pairs of considerably reduced size in the general case.

\section{Preliminaries}

We start with a brief introduction of $\mathcal{M}$-adhesive categories, rules with nested application conditions (ACs) (cf. Subsect.3.2), and the main parts of critical pair theory for this type of rules [6,9] (cf. Subsect.3.3). Thereafter, we reintroduce the notion of initial conflicts [19] for plain rules, i.e. rules without ACs (cf. Subsect.3.4). We also introduce the notion of initial parallel independent transformation pairs as a counterpart (cf. Subsect. 3.5), since it will play a particular role when defining initial conflicts for rules with ACs in Subsect. 3.4. We assume that the reader is acquainted with the basic theory of DPO graph transformation and, in particular, the standard definitions of typed graphs and typed graph morphisms (see, e.g., [5]) and its associated category, Graphs $\mathbf{G G}_{T}$.

\subsection{Graphs and High-Level Structures}

Our results do not only apply to a specific class of graph transformation systems, like standard (typed) graph transformation systems, but to systems over any $\mathcal{M}$-adhesive category [5]. The idea behind considering $\mathcal{M}$-adhesive categories is to avoid similar investigations for different instantiations like e.g. Petri nets or hypergraphs. An $\mathcal{M}$-adhesive category is a category $\mathcal{C}$ with a distinguished morphism class $\mathcal{M}$ of monomorphisms satisfying certain properties. The most important one is the van Kampen (VK) property stating a certain kind of compatibility of pushouts and pullbacks along $\mathcal{M}$-morphisms. Moreover, additional properties are needed in our context: initial pushouts, describing the existence of a special "smallest" pushout over a morphism, $\mathcal{E}^{\prime}-\mathcal{M}$ pair factorizations, extending the classical epi-mono factorization to a pair of morphisms with the same codomain. The definitions of these properties can be found in $[6,7]$.

Assumption 1. We assume that $\langle\mathcal{C}, \mathcal{M}\rangle$ is an $\mathcal{M}$-adhesive category with a unique $\mathcal{E}^{\prime}-\mathcal{M}$ pair factorization (needed for Lemma 1, Definition 5, Theorem 3, Corollary 1) and binary coproducts (needed for Lemma3, Definition8, Theorem 1). For the Local Confluence Theorem for initial conflicts of rules with ACs we in addition need initial pushouts (cf. Subsect. 4.4).

Remark 1. Most categories of structures used for specification are $\mathcal{M}$-adhesive and satisfy these additional properties [5], including the category $\left\langle\operatorname{Graphs}_{T G}, \mathcal{M}\right\rangle$ with $\mathcal{M}$ being the class of all injective typed graph morphisms. 


\subsection{Rules with Application Conditions and Parallel Independence}

Nested application conditions [11] (in short, application conditions, or just ACs) generalize the corresponding notions in $[4,10,15]$, where a negative (positive) application condition, short NAC (PAC), over a graph $P$, denoted $\neg \exists a(\exists a)$ is defined in terms of a morphism $a: P \rightarrow C$. Informally, a morphism $m: P \rightarrow G$ satisfies $\neg \exists a(\exists a)$ if there does not exist a morphism $q: C \rightarrow G$ extending $a$ to $m$ (if there exists $q$ extending $a$ to $m$ ). Then, an AC is either the special condition true or a pair of the form $\exists\left(a, \mathrm{ac}_{C}\right)$ or $\neg \exists\left(a, \mathrm{ac}_{C}\right)$, where $\mathrm{ac}_{C}$ is an additional AC on $C$. Intuitively, a morphism $m: P \rightarrow G$ satisfies $\exists\left(a, a_{C}\right)$ if $m$ satisfies $a$ and the corresponding extension $q$ satisfies $\mathrm{ac}_{C}$. Moreover, ACs may be combined with the usual logical connectors. For a concrete definition of ACs we address the reader to [11] or [6].

ACs are used to restrict the application of rules to a given object. The idea is to equip the precondition (or left hand side) of rules with an application condition. Then we can only apply a given rule to an object $G$ if the corresponding match morphism satisfies the $\mathrm{AC}$ of the rule. However, for technical reasons, we also introduce the application of rules disregarding the associated ACs.

Definition 1 (rules and transformations). A rule $\rho=\left\langle p, \mathrm{ac}_{L}\right\rangle$ consists of a plain rule $p=\langle L \hookleftarrow I \rightarrow R\rangle$ with $I \hookrightarrow L$ and $I \hookrightarrow R$ morphisms in $\mathcal{M}$ and an application condition $\mathrm{ac}_{L}$ over $L$.

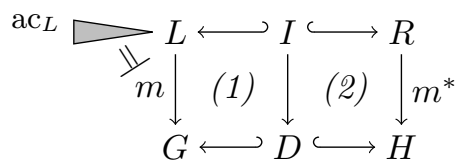

$A$ direct transformation $t: G \Rightarrow_{\rho, m, m^{*}} H$ consists of two pushouts (1) and (2), called DPO, with match $m$ and comatch $m^{*}$ such that $m \models \operatorname{ac}_{L} . G \hookleftarrow D \hookrightarrow$ $H$ is called the derived span of $t$. An AC-disregarding direct transformation $G \Rightarrow_{\rho, m, m^{*}} H$ consists of DPO (1) and (2), where $m$ does not necessarily need to satisfy $\operatorname{ac}_{L}$. Given a set of rules $\mathcal{R}$ for $\langle\mathcal{C}, \mathcal{M}\rangle$, the triple $\langle\mathcal{C}, \mathcal{M}, \mathcal{R}\rangle$ is an $\mathcal{M}$-adhesive system.

Remark 2. In the rest of the paper we assume that each rule (resp. transformation or $\mathcal{M}$-adhesive system) comes with ACs. Otherwise, we state that we have a plain rule (resp. transformation or $\mathcal{M}$-adhesive system), which can be seen as a special case, in the sense that the ACs are (equivalent to) true.

ACs can be shifted over morphisms and rules as shown in the following lemma (for constructions see $[7]^{2}$ and $[7,11]$, respectively).

\footnotetext{
${ }^{2}$ Since this construction entails the enumeration of jointly epimorphic morphism pairs, its computation has exponential complexity in the size of the precondition of the rule and the size of the AC.
} 
Lemma 1 (shift ACs over morphisms [7]). There is a transformation Shift from morphisms and $A C s$ to $A C s$ such that for each $A C, \mathrm{ac}_{P}$, and each morphism $b: P \rightarrow P^{\prime}$, Shift transforms $\operatorname{ac}_{P}$ via $b$ into an $A C \operatorname{Shift}\left(b, \operatorname{ac}_{P}\right)$ over $P^{\prime}$ such that for each morphism $n: P^{\prime} \rightarrow H$ it holds that $n \circ b=\operatorname{ac}_{P} \Leftrightarrow n \models \operatorname{Shift}\left(b, \operatorname{ac}_{P}\right)$.

Lemma 2 (shift ACs over rules [7,11]). There is a transformation L from rules and $A C s$ to $A C s$ such that for every rule $\rho: L \hookleftarrow I \hookrightarrow R$ and every $A C$ on $R, \mathrm{ac}_{R}$, L transforms $\mathrm{ac}_{R}$ via $\rho$ into the $A C \mathrm{~L}\left(\rho, \mathrm{ac}_{R}\right)$ on $L$, such that for every direct transformation $G \Rightarrow_{\rho, m, m^{*}} H, m=\mathrm{L}\left(\rho, \mathrm{ac}_{R}\right) \Leftrightarrow m^{*}=\mathrm{ac}_{R}$.

For parallel independence, when working with rules with ACs, we need not only that each rule does not delete any element which is part of the match of the other rule, but also that the resulting transformation defined by each rule application still satisfies the ACs of the other rule application.

Definition 2 (transformation pairs and parallel independence). $A$ transformation pair $H_{1} \Leftarrow_{\rho_{1}, o_{1}} G \Rightarrow_{\rho_{2}, o_{2}} H_{2}$ is parallel independent if there exists a morphism $d_{12}: L_{1} \rightarrow D_{2}$ such that $k_{2} \circ d_{12}=o_{1}$ and $c_{2} \circ d_{12} \models \mathrm{ac}_{L_{1}}$ and there exists a morphism $d_{21}: L_{2} \rightarrow D_{1}$ such that $k_{1} \circ d_{21}=o_{2}$ and $c_{1} \circ d_{21} \models \operatorname{ac}_{L_{2}}$.

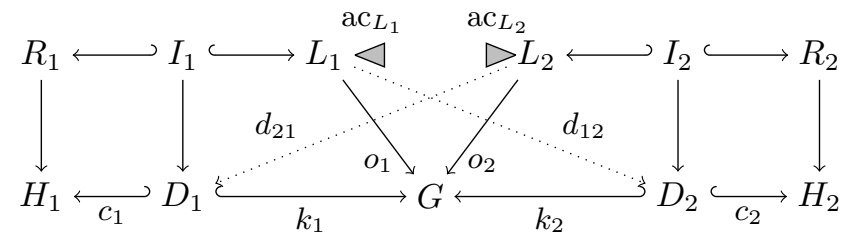

A transformation pair is in conflict or conflicting if it is parallel dependent. We distinguish different conflict types, generalizing straightforwardly the conflict characterization introduced for rules with NACs [20]. The transformation pair $H_{1} \Leftarrow_{\rho_{1}, o_{1}} G \Rightarrow_{\rho_{2}, o_{2}} H_{2}$ is a use-delete (resp. delete-use) conflict if in Definition 2 the commuting morphism $d_{12}$ (resp. $d_{21}$ ) does not exist, i.e. the second (resp. first) rule deletes something used by the first (resp. second) one. Moreover, it is an $A C$-produce (resp. produce- $A C$ ) conflict if in Definition 2 the commuting morphism $d_{12}$ (resp. $d_{21}$ ) exists, but an extended match is produced by the second (resp. first) rule that does not satisfy the rule $\mathrm{AC}$ of the first (resp. second) rule. If a transformation pair is an $A C$-produce or produce- $A C$ conflict, then we also say that it is an $A C$ conflict or $A C$ conflicting.

Remark 3 (use-delete XOR AC-produce). A use-delete (resp. delete-use) conflict cannot occur simultaneously to an AC-produce (resp. produce-AC) conflict, since the $\mathrm{AC}$ of the first (resp. second) rule can only be violated iff there exists an extended match for the first (resp. second) rule. However, a use-delete (resp. delete-use) conflict may occur simultaneously to a produce-AC (resp. ACproduce) conflict.

For grasping the notion of completeness of transformation pairs w.r.t. a property like parallel (in-)dependence, it is first important to understand how a given 
transformation can be extended to another transformation. In particular, an extension diagram describes how a transformation $t: G_{0} \Rightarrow^{*} G_{n}$ can be extended to a transformation $t^{\prime}: G_{0}^{\prime} \Rightarrow^{*} G_{n}^{\prime}$ via the same rules and an extension morphism $k_{0}: G_{0} \rightarrow G_{0}^{\prime}$ that maps $G_{0}$ to $G_{0}^{\prime}$ as shown in the following diagram on the left. For each rule application and transformation step, we have two double pushout diagrams as shown on the right, where the rule $\rho_{i+1}$ is applied to $G_{i}$ and $G_{i}^{\prime}$.
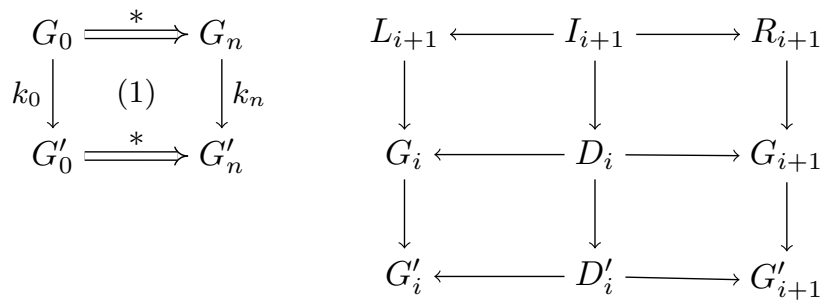

We introduce two notions of completeness, distinguishing $\mathcal{M}$-completeness from regular completeness, depending on the membership of the extension morphism in $\mathcal{M}$. It is known that critical pairs (resp. initial conflicts) for plain rules are $\mathcal{M}$-complete (resp. complete) w.r.t. parallel dependence $[5,19]$. In Subsect.3.3, we reintroduce the fact that critical pairs for rules with ACs are $\mathcal{M}$-complete w.r.t. parallel dependence, but as symbolic transformation pairs. We learn in Sect. 4 that initial conflicts for rules with ACs are also complete in this symbolic way.

Definition 3 ((M-)completeness of transformation pairs). A set of transformation pairs $\mathcal{S}$ for a pair of rules $\left\langle\rho_{1}, \rho_{2}\right\rangle$ is complete (resp. $\mathcal{M}$ complete) w.r.t. parallel (in-)dependence if there is a pair $P_{1} \Leftarrow{ }_{\rho_{1}, o_{1}} K \Rightarrow_{\rho_{2}, o_{2}} P_{2}$ from $\mathcal{S}$ and an extension diagram via extension morphism $m$ (resp. $m \in \mathcal{M}$ ) for each parallel (in-)dependent direct transformation pair $H_{1} \Leftarrow \rho_{1}, m_{1} G \Rightarrow_{\rho_{2}, m_{2}} H_{2}$.

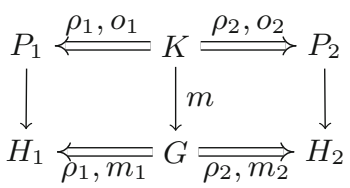

Fig. 1. (M-)completeness of transformation pairs

\subsection{Critical Pairs}

Critical pairs for plain rules are just transformation pairs, where morphisms $o_{1}$ and $o_{2}$ are in $\mathcal{E}^{\prime}$ (i.e., roughly, $K$ is an overlapping of $L_{1}$ and $L_{2}$ ). In the category of Graphs they lead to finite and complete subsets of finite conflicts [4] (assumed 
that the rule graphs are also finite). However, when rules include ACs, we cannot use the same notion of critical pair since, as we show in Theorem 2, in general, for any two rules with ACs, there is no complete set of transformation pairs that is finite. To avoid this problem, our critical pairs for rules with ACs also include $\mathrm{ACs}$, as in $[6,9]$, where they are proved to be $\mathcal{M}$-complete, and they are also finite in the category of Graphs (assumed again that the rules are finite).

In particular, critical pairs are based on the notion of symbolic transformation pairs, which are pairs of $A C$-disregarding transformations on some object $K$ with two special ACs on $K$. These two $\mathrm{ACs}, \mathrm{ac}_{K}$ (extension $A C$ ) and $\mathrm{ac}_{K}^{*}$ (conflict-inducing $A C$ ), are used to characterize which embeddings of this pair, via some morphism $m: K \rightarrow G$, give rise to a transformation pair that is parallel dependent. If $m \models \operatorname{ac}_{K}$, then $m \circ o_{1}: L_{1} \rightarrow G$ and $m \circ o_{2}: L_{2} \rightarrow G$ are two morphisms, satisfying the associated ACs of $\rho_{1}$ and $\rho_{2}$, respectively. Moreover, if $m \models \mathrm{ac}_{K}^{*}$, then the two transformations $H_{1} \Leftarrow_{\rho_{1}, m \circ o_{1}} G \Rightarrow_{\rho_{2}, m \circ o_{2}} H_{2}$ are parallel dependent. Symbolic transformation pairs allow us to present critical pairs as well as initial conflicts (cf. Subsect.3.4) in a compact and unified way, since they both are instances of symbolic transformation pairs. Finally, note that each symbolic transformation pair $s t p_{K}:\left\langle t p_{K}, a c_{K}, a c_{K}^{*}\right\rangle$ is by definition uniquely determined (up to isomorphism and equivalence of the extension $\mathrm{AC}$ and conflictinducing AC) by its underlying AC-disregarding transformation pair.

Definition 4 (symbolic transformation pair). Given rules $\rho_{1}=\left\langle p_{1}\right.$, ac $\left._{L_{1}}\right\rangle$ and $\rho_{2}=\left\langle p_{2}, \operatorname{ac}_{L_{2}}\right\rangle$, a symbolic transformation pair $s t p_{K}:\left\langle t p_{K}, a c_{K}, a c_{K}^{*}\right\rangle$ for $\left\langle\rho_{1}, \rho_{2}\right\rangle$ consists of a pair tp $p_{K}: P_{1} \Leftarrow \rho_{1}, o_{1} \quad K \Rightarrow_{\rho_{2}, o_{2}} P_{2}$ of AC-disregarding transformations together with $A C s \mathrm{ac}_{K}$ and $\mathrm{ac}_{K}^{*}$ on $K$ given by:

$\operatorname{ac}_{K}=\operatorname{Shift}\left(o_{1}, \operatorname{ac}_{L_{1}}\right) \wedge \operatorname{Shift}\left(o_{2}, \operatorname{ac}_{L_{2}}\right)$, called extension $\mathrm{AC}$, and

$\mathrm{ac}_{K}^{*}=\neg\left(\mathrm{ac}_{K, d_{12}}^{*} \wedge \mathrm{ac}_{K, d_{21}}^{*}\right)$, called conflict-inducing $\mathrm{AC}$

with $\operatorname{ac}_{K, d_{12}}^{*}$ and $\mathrm{ac}_{K, d_{21}}^{*}$ given as follows:

$$
\begin{gathered}
\text { if }\left(\exists d_{12} \text { with } k_{2} \circ d_{12}=o_{1}\right) \text { then } \operatorname{ac}_{K, d_{12}}^{*}=\mathrm{L}\left(p_{2}^{*}, \operatorname{Shift}\left(c_{2} \circ d_{12}, \operatorname{ac}_{L_{1}}\right)\right) \\
\qquad \begin{aligned}
& \text { else } \operatorname{ac}_{K, d_{12}}^{*}=\text { false } \\
& \text { if }\left(\exists d_{21} \text { with } k_{1} \circ d_{21}=o_{2}\right) \text { then } \operatorname{ac}_{K, d_{21}}^{*}=\mathrm{L}\left(p_{1}^{*}, \operatorname{Shift}\left(c_{1} \circ d_{21}, \operatorname{ac}_{L_{2}}\right)\right) \\
& \text { else } \operatorname{ac}_{K, d_{21}}^{*}=\text { false }
\end{aligned}
\end{gathered}
$$

where $p_{1}^{*}=\left\langle K \stackrel{k_{1}}{\longleftrightarrow} D_{1} \stackrel{c_{1}}{\hookrightarrow} P_{1}\right\rangle$ and $p_{2}^{*}=\left\langle K \stackrel{k_{2}}{\longleftrightarrow} D_{2} \stackrel{c_{2}}{\hookrightarrow} P_{2}\right\rangle$ are defined by the corresponding double pushouts.

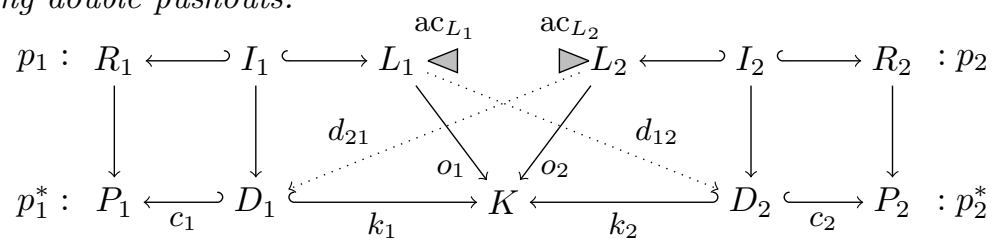

A critical pair is now a symbolic transformation pair in a minimal context such that there exists at least one extension to a pair of transformations being parallel dependent. 
Definition 5 (critical pair). Given rules $\rho_{1}=\left\langle p_{1}, \mathrm{ac}_{L_{1}}\right\rangle$ and $\rho_{2}=\left\langle p_{2}\right.$, $\left.\mathrm{ac}_{L_{2}}\right\rangle$, a critical pair for $\left\langle\rho_{1}, \rho_{2}\right\rangle$ is a symbolic transformation pair $\operatorname{stp}_{K}:\left\langle t p_{K}\right.$, $\left.\mathrm{ac}_{K}, \mathrm{ac}_{K}^{*}\right\rangle$, where the match pair $\left(o_{1}, o_{2}\right)$ of $t p_{K}$ is in $\mathcal{E}^{\prime}$, and there exists a morphism $m: K \rightarrow G \in \mathcal{M}$ such that $m=\operatorname{ac}_{K} \wedge \operatorname{ac}_{K}^{*}$ and $m_{i}=m \circ o_{i}$, for $i=1,2$, satisfy the gluing conditions, i.e. $m_{i}$ has a pushout complement w.r.t. $p_{i}$.

Definition 6 ((M-)completeness of symbolic transformation pairs). $A$ set of symbolic transformation pairs $\mathcal{S}$ for a pair of rules $\left\langle\rho_{1}, \rho_{2}\right\rangle$ is complete (resp. $\mathcal{M}$-complete) w.r.t. parallel dependence if there is a symbolic transformation pair stp $p_{K}:\left\langle t p_{K}: P_{1} \Leftarrow_{\rho_{1}, o_{1}} K \Rightarrow_{\rho_{2}, o_{2}} P_{2}, \mathrm{ac}_{K}, \mathrm{ac}_{K}^{*}\right\rangle$ from $\mathcal{S}$ and an extension diagram as depicted in Fig. 1 with $m: K \rightarrow G$ (resp. $m: K \rightarrow$ $G \in \mathcal{M})$ and $m \models \operatorname{ac}_{K} \wedge \mathrm{ac}_{K}^{*}$ for each parallel dependent direct transformation $H_{1} \Leftarrow_{\rho_{1}, m_{1}} G \Rightarrow_{\rho_{2}, m_{2}} H_{2}$.

In $[6,9]$ it is shown that the set of critical pairs for a pair of rules is $\mathcal{M}$ complete w.r.t. parallel dependence.

\subsection{Initial Conflicts for Plain Rules}

Initial conflicts for plain rules follow an alternative approach to the original idea of critical pairs. Instead of considering all conflicting transformations in a minimal context (materialized by a pair of jointly epimorphic matches), initial conflicts use the notion of initiality of transformation pairs to obtain a more declarative view on the minimal context of critical pairs. Each initial conflict is a critical pair but not the other way round. Moreover, all initial conflicts for plain rules are complete w.r.t. parallel dependence and they still satisfy the Local Confluence Theorem for plain rules. Consequently, initial conflicts for plain rules represent an important, proper subset of critical pairs for performing static conflict detection as well as local confluence analysis.

Definition 7 (initial transformation pair and initial conflict). Given a pair of plain direct transformations $t p: H_{1} \Leftarrow_{p_{1}, m_{1}} G \Rightarrow_{p_{2}, m_{2}} H_{2}$, then $t p^{I}$ : $H_{1}^{I} \Leftarrow_{p_{1}, m_{1}^{I}} G^{I} \Rightarrow_{p_{2}, m_{2}^{I}} H_{2}^{I}$ is an initial transformation pair for tp if it can be embedded into tp via extension diagrams (1) and (2) and extension morphism $f^{I}$, as in the left diagram below, such that for each transformation pair $t p^{\prime}$ : $H_{1}^{\prime} \Leftarrow_{p_{1}, m_{1}^{\prime}} G^{\prime} \Rightarrow_{p_{2}, m_{2}^{\prime}} H_{2}^{\prime}$ that can be embedded into tp via extension diagrams (3) and (4) and extension morphism $f$, as in the left diagram below, it holds that $t p^{I}$ can be embedded into t $p^{\prime}$ via unique extension diagrams (5) and (6) and unique vertical morphism $f^{\prime I}$ s.t. $f \circ f^{I}=f^{I}$.

Given a plain $\mathcal{M}$-adhesive system with initial transformation pairs for conflicts, an initial conflict is a conflict tp isomorphic to $t p^{I}$. 


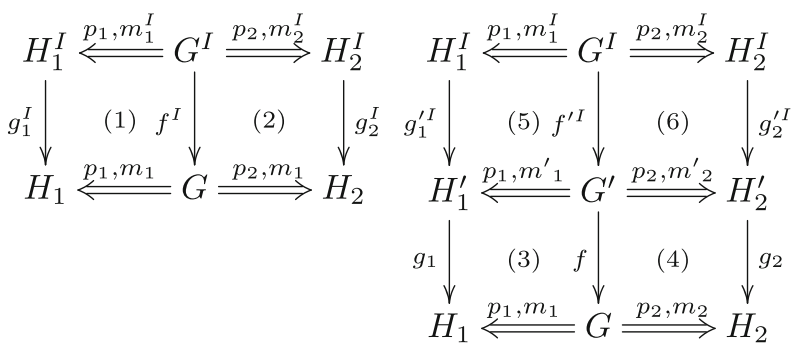

The idea of representing all conflicts by a (finite) set of initial conflicts is based on the requirement of the existence of initial transformation pairs for parallel dependent or conflicting plain transformation pairs. This requirement holds for the category of typed graphs [19] and for any arbitrary $\mathcal{M}$-adhesive category fulfilling some extra conditions [1].

For plain $\mathcal{M}$-adhesive systems, initial conflicts are critical pairs [19]. Moreover, they are complete and minimal as transformation pairs w.r.t. parallel dependence, whereas critical pairs for plain rules are $\mathcal{M}$-complete [4].

\subsection{Initial Parallel Independent Transformation Pairs for Plain Rules}

In this section, we show the existence of initial transformation pairs for parallel independent transformation pairs (Fig. 2), allowing us to define a complete subset also w.r.t. parallel independence. The proof requires that binary coproducts exist.

Lemma 3 (existence of initial transformation pair for parallel independent transformation pair). Given a pair of parallel independent plain direct transformations $t p: H_{1} \Leftarrow_{p_{1}, m_{1}} G \Rightarrow_{p_{2}, m_{2}} H_{2}$, then $t_{L_{1}+L_{2}}: R_{1}+$ $L_{2} \Leftarrow_{p_{1}, i_{1}} L_{1}+L_{2} \Rightarrow_{p_{2}, i_{2}} L_{1}+R_{2}$, where $i_{1}: L_{1} \rightarrow L_{1}+L_{2}$ and $i_{2}: L_{2} \rightarrow L_{1}+L_{2}$ are the coproduct morphisms, is initial for tp.

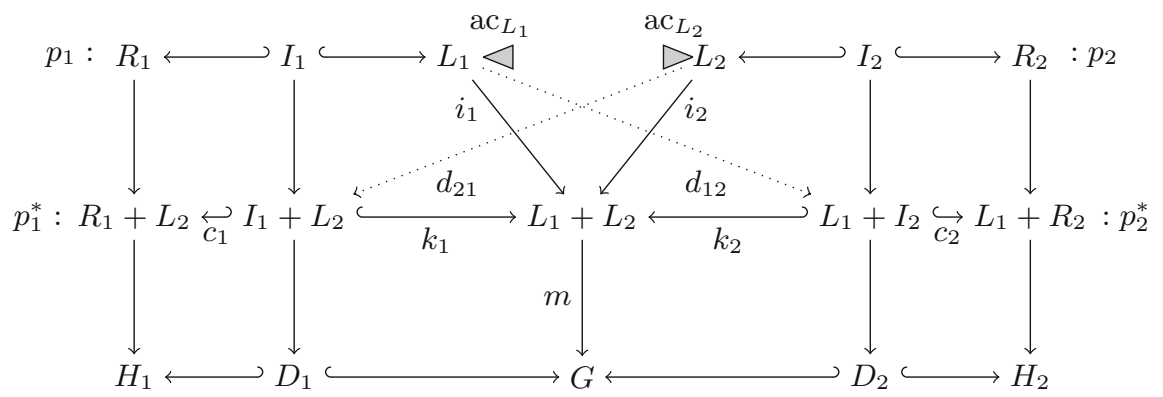

Fig. 2. Initial parallel independent transformation pair $t p_{L_{1}+L_{2}}$ 
Uniqueness of initial transformation pairs up to isomorphism implies that for each pair of plain rules $\left\langle p_{1}, p_{2}\right\rangle$ there is a unique initial parallel independent transformation pair $\operatorname{tp}_{L_{1}+L_{2}}: R_{1}+L_{2} \Leftarrow_{p_{1}, i_{1}} L_{1}+L_{2} \Rightarrow_{p_{2}, i_{2}} L_{1}+R_{2}$.

Definition 8 (initial parallel independent transformation pair). A pair of parallel independent plain transformations $t p: H_{1} \Leftarrow_{p_{1}, m_{1}} G \Rightarrow_{p_{2}, m_{2}} H_{2}$ is an initial parallel independent transformation pair if it is isomorphic to the transformation pair $\operatorname{tp}_{L_{1}+L_{2}}: R_{1}+L_{2} \Leftarrow_{p_{1}, i_{1}} L_{1}+L_{2} \Rightarrow_{p_{2}, i_{2}} L_{1}+R_{2}$.

The one-element set consisting of the initial parallel independent transformation pair for a given pair of rules is complete w.r.t. parallel independence.

Theorem 1 (completeness of initial parallel independent transformation pairs). The set consisting of the initial parallel independent transformation pair tp $_{L_{1}+L_{2}}: R_{1}+L_{2} \Leftarrow_{p_{1}, i_{1}} L_{1}+L_{2} \Rightarrow_{p_{2}, i_{2}} L_{1}+R_{2}$ for a pair of plain rules $\left\langle p_{1}, p_{2}\right\rangle$ is complete w.r.t. parallel independence.

\section{Initial Conflicts}

We start with showing why it is not possible to straightforwardly generalize the idea of initial conflicts from plain rules to rules with ACs. On the one hand, conflict inheritance, which was the basis for showing completeness of initial conflicts for plain rules, does not hold any more. Moreover, it is impossible in general to find a finite and complete subset of finite conflicts for rules with ACs (cf. Subsect.4.2). This motivates again the need for having symbolic transformation pairs as introduced in Definition 4, allowing us to define initial conflicts (cf. Subsect. 4.3) as a set of specific symbolic transformation pairs, being complete w.r.t. parallel dependence indeed (as shown in Subsect.4.4).

\subsection{Conflict Inheritance}

Conflicts are in general not inherited (as opposed to the case of plain rules [19]), i.e., not each (initial) transformation pair that can be embedded into a conflicting one will be conflicting again. This may happen in particular for AC conflicts. Use-delete (resp. delete-use) conflicts for rules with ACs are still inherited.

Lemma 4 (Use-delete (delete-use) conflict inheritance). Given a pair of direct transformations tp in use-delete (resp. delete-use) conflict and another pair of direct transformations t $p^{\prime}$ that can be embedded into tp via extension morphism $f$ and corresponding extension diagrams, then $t p^{\prime}$ is also in use-delete (resp. delete-use) conflict.

Example 1 (No inheritance for $A C$ conflicts). Consider rules $p_{1}: \bigcirc \leftarrow \bigcirc \rightarrow$ $\bigcirc \rightarrow \bigcirc$ (with AC true), producing an outgoing edge with a node, and $p_{2}: \bigcirc \leftarrow$ $\bigcirc \rightarrow \bigcirc \bigcirc$ with NAC $\neg \exists n: \bigcirc \rightarrow \bigcirc \bigcirc \bigcirc$, producing a node only if two other nodes do not exist already. Consider graph $G=\bigcirc \bigcirc$, holding two nodes. Applying both 
rules to $G$ (with the matches sharing one node in $G$ ) we obtain a produce-AC conflict since the first rule creates a third node, forbidden by the second rule. Now both rules can be applied similarly to the shared node in the subgraph $G^{\prime}=\bigcirc$ of $G$ obtaining parallel independent transformations, illustrating that AC-conflicts are not inherited.

\subsection{Complete Subset of Conflicts}

We show that in $\mathcal{M}$-adhesive categories, in particular in the category of graphs, it is in general impossible to find a finite and complete subset of conflicts for finite rules with ACs. If it would always exist, we could derive that each first-order formula is equivalent to a finite disjunction of atomic formulas.

Theorem 2. Given finite rules $\rho_{1}=\left\langle p_{1}, \mathrm{ac}_{L_{1}}\right\rangle$ and $\rho_{2}=\left\langle p_{2}, \mathrm{ac}_{L_{2}}\right\rangle$ for the $\mathcal{M}$-adhesive category Graphs, in general, there is no finite set of finite transformation pairs $\mathcal{S}$ for $\rho_{1}$ and $\rho_{2}$ that is complete w.r.t. parallel dependence.

\subsection{Initial Conflicts}

We generalize the notion of initial conflicts for plain rules to rules with ACs. In particular, we introduce them as special symbolic transformation pairs. They are conflict-inducing meaning that there needs to exist an unfolding of the symbolic transformation pair into a concrete conflicting transformation pair. Moreover, their AC-disregarding transformation pair needs to be an initial conflict or initial parallel independent transformation pair. We also show the relationship between initial conflicts and critical pairs as reintroduced in Subsect. 3.3, demonstrating that initial conflicts represent a proper subset of critical pairs.

Definition 9 (unfolding of symbolic transformation pair). Given a symbolic transformation pair st $p_{K}:\left\langle t p_{K}, \mathrm{ac}_{K}, \mathrm{ac}_{K}^{*}\right\rangle$ for rule pair $\left\langle\rho_{1}, \rho_{2}\right\rangle$, then its unfolding $\mathcal{U}\left(\operatorname{stp}_{K}\right)$ consists of all transformation pairs $H_{1} \Leftarrow \rho_{1}, m_{1} G \Rightarrow_{\rho_{2}, m_{2}} H_{2}$ representing the lower row of the extension diagrams via some extension morphism $m: K \rightarrow G$ as shown in Fig. 1 (with AC-disregarding transformation pair $t p_{K}$ in the upper row). Moreover, we say that $s t p_{K}$ is conflict-inducing if its unfolding includes a conflicting transformation pair.

Definition 10 (initial conflict). Consider an $\mathcal{M}$-adhesive system with initial transformation pairs for conflicts along plain rules. An initial conflict for rules $\rho_{1}=\left\langle p_{1}, \operatorname{ac}_{L_{1}}\right\rangle$ and $\rho_{2}=\left\langle p_{2}, \operatorname{ac}_{L_{2}}\right\rangle$ is a conflict-inducing symbolic transformation pair $\operatorname{stp}_{K}:\left\langle t p_{K}, \mathrm{ac}_{K}, \mathrm{ac}_{K}^{*}\right\rangle$ with the AC-disregarding transformation pair $t p_{K}$ being initial, i.e. either $t p_{K}$ is an initial conflict for rules $p_{1}$ and $p_{2}$ (in this case $\operatorname{stp}_{K}$ is called a use-delete/delete-use initial conflict) or it is the initial parallel independent transformation pair $t p_{L_{1}+L_{2}}$ for rules $p_{1}$ and $p_{2}$ (in this case $\operatorname{stp}_{K}=\operatorname{stp}_{L_{1}+L_{2}}=\left\langle t p_{L_{1}+L_{2}}, \mathrm{ac}_{L_{1}+L_{2}}, \mathrm{ac}_{L_{1}+L_{2}}^{*}\right\rangle$ is called the AC initial conflict). 
Note that the unfolding of a conflict-inducing symbolic transformation pair (and in particular of an AC initial conflict) may also include parallel independent transformation pairs. All conflicts in the unfolding of an AC initial conflict are $\mathrm{AC}$ conflicts, and never use-delete/delete-use conflicts (because otherwise we would get a contradiction using Lemma 4).

Example 2 (initial conflict). Consider again the rules from Example 1. Applying both rules to $L_{1}+L_{2}=\bigcirc \bigcirc$ (with disjoint matches) we obtain the AC initial conflict $\operatorname{stp}_{K}=s t p_{L_{1}+L_{2}}=\left\langle t p_{L_{1}+L_{2}}, \mathrm{ac}_{L_{1}+L_{2}}, \mathrm{ac}_{L_{1}+L_{2}}^{*}\right\rangle$. Thereby $\mathrm{ac}_{L_{1}+L_{2}}$ is equivalent to $\neg \exists\left(\mathrm{O}_{1} \mathrm{O}_{2} \rightarrow \mathrm{O}_{1} \mathrm{O}_{2} \bigcirc\right) \wedge \neg \exists\left(\mathrm{O}_{1} \mathrm{O}_{2} \rightarrow \mathrm{O}_{1,2} \bigcirc \mathrm{O}\right)$, expressing that when during extension both nodes are merged, no two additional nodes, otherwise not one additional node should be given. Moreover, $\mathrm{ac}_{L_{1}+L_{2}}^{*}$ is equivalent to $\exists\left(\bigcirc_{1} \bigcirc_{2} \rightarrow \bigcirc_{1,2} \bigcirc\right) \vee \exists\left(\bigcirc_{1} \bigcirc_{2} \rightarrow \bigcirc_{1} \bigcirc_{2}\right)$, expressing that either both nodes are not merged during extension, otherwise one additional node should be present for a conflict to arise. Both transformation pairs (the conflicting one from $G=\bigcirc \bigcirc$ as well as the parallel independent one from its subgraph $G^{\prime}=0$, sharing the merged node in their matches) described in Example 1 belong to its unfolding.

Each initial conflict is in particular also a critical pair.

Theorem 3 (initial conflict is critical pair). Consider an $\mathcal{M}$-adhesive system with initial transformation pairs for conflicts along plain rules. Each initial conflict $\operatorname{stp}_{K}:\left\langle t p_{K}, \mathrm{ac}_{K}, \mathrm{ac}_{K}^{*}\right\rangle$ is a critical pair.

The reverse direction of Theorem 3 does not hold. In general, critical pairs $s t p_{K}:\left\langle t p_{K}, \mathrm{ac}_{K}, \mathrm{ac}_{K}^{*}\right\rangle$ where $t p_{K}$ represents a use-delete/delete-use conflict (but is not initial yet) are represented by the initial conflict $s t p_{I}:\left\langle t p_{I}, \mathrm{ac}_{I}, \mathrm{ac}_{I}^{*}\right\rangle$ with $t p_{I}$ the unique initial conflict for $t p_{K}$ as plain transformation pair. Moreover, critical pairs $s t p_{K}:\left\langle t p_{K}, \mathrm{ac}_{K}, \mathrm{ac}_{K}^{*}\right\rangle$ where $t p_{K}$ is parallel independent as plain transformation pair are represented by one initial conflict $s t p_{L_{1}+L_{2}}$ : $\left\langle t p_{L_{1}+L_{2}}, \operatorname{ac}_{L_{1}+L_{2}}, \mathrm{ac}_{L_{1}+L_{2}}^{*}\right\rangle$ with $t p_{L_{1}+L_{2}}$ the initial parallel independent transformation pair.

Example 3 (initial conflicts: proper subset of critical pairs). Consider again the rules from Example 1 and their application to $G^{\prime}=0$. The symbolic transformation pair $\operatorname{stp}_{G^{\prime}}:\left\langle t p_{G^{\prime}}, \operatorname{ac}_{G^{\prime}}\right.$, ac $\left._{G^{\prime}}^{*}\right\rangle$ is a critical pair, but not an initial conflict.

\subsection{Completeness}

We show that initial conflicts are complete (not $\mathcal{M}$-complete as in the case of critical pairs) w.r.t. parallel dependence as symbolic transformation pairs.

Theorem 4 (completeness of initial conflicts). Consider an $\mathcal{M}$-adhesive system with initial transformation pairs for conflicts along plain rules. The set of initial conflicts for a pair of rules $\left\langle\rho_{1}, \rho_{2}\right\rangle$ is complete w.r.t. parallel dependence. 
Remark 4 (uniqueness of initial conflicts). For each conflict a unique (up-toisomorphism) initial conflict exists representing it, since this property is inherited from the one for plain rules [19] and the fact that the initial parallel independent pair of transformations is unique w.r.t. a given rule pair.

Initial conflicts are also minimally complete, i.e. we are able to generalize the corresponding result for plain rules to rules with ACs.

\section{Unfoldings of Initial Conflicts}

We show a sufficient condition for being able to unfold initial conflicts into a complete set of conflicts that is finite if the set of initial conflicts is finite (cf. Subsect. 5.1). We demonstrate moreover that this sufficient condition is fulfilled for the special case of having merely NACs as rule application conditions (cf. Subsect.5.2). Finally, we show that in this case we obtain in particular specific critical pairs for rules with negative application conditions (NACs) as introduced in [20] again. In this sense we show explicitly that initial conflicts as introduced in this paper represent a conservative extension of the critical pair theory for rules with NACs.

\subsection{Finite and Complete Unfolding}

We introduce regular initial conflicts leading to $\mathcal{M}$-complete subsets of conflicts by unfolding them in some particular way (cf. disjunctive unfolding in Definition 11). The idea is that the extension and conflict-inducing $\mathrm{AC}\left(\mathrm{ac}_{K}\right.$ and $\mathrm{ac}_{K}^{*}$, respectively) of such a conflict $s t p_{K}:\left\langle t p_{K}, \mathrm{ac}_{K}, \mathrm{ac}_{K}^{*}\right\rangle$ have a specific form that is amenable to finding $\mathcal{M}$-complete unfoldings. We expect the condition $\operatorname{ac}_{K} \wedge \mathrm{ac}_{K}^{*}$ to consist of a disjunction of positive literals (conditions of the form $\exists\left(a_{i}: K \rightarrow C_{i}, c_{i}\right)$ ) with a negative remainder (i.e. a condition $\left.c_{i}=\wedge_{j \in J} \neg \exists\left(b_{j}: C_{i} \rightarrow C_{j}, d_{j}\right)\right)$. Intuitively, this means that there is a finite number of possibilities to unfold the symbolic conflict into a concrete conflict by adding some specific positive context (expressed by the morphism $a_{i}$ ). The negative remainder $c_{i}$ ensures that by adding this positive context to the context $K$ of the symbolic transformation pair within the initial conflict, we indeed find a concrete conflict when not extending further at all. Moreover, it expresses under which condition the corresponding concrete representative conflict leads to further conflicts by extension. Finally, the subsets of $\mathcal{M}$-complete conflicts built using the disjunctive unfolding are finite if the set of initial conflicts it is derived from is finite.

Definition 11 (regular initial conflict, disjunctive unfolding). Consider an $\mathcal{M}$-adhesive system with initial transformation pairs for conflicts along plain rules. Given an initial conflict $\operatorname{stp}_{K}:\left\langle t p_{K}, \mathrm{ac}_{K}, \operatorname{ac}_{K}^{*}\right\rangle$ for rules $\left\langle\rho_{1}, \rho_{2}\right\rangle$, then we say that it is regular if $\operatorname{ac}_{K} \wedge \mathrm{ac}_{K}^{*}$ is equivalent to a condition $\vee_{i \in I} \exists\left(a_{i}\right.$ : $\left.K \rightarrow C_{i}, c_{i}\right)$ with $c_{i}=\wedge_{j \in J} \neg \exists\left(b_{j}: C_{i} \rightarrow C_{j}, d_{j}\right)$ a condition on $C_{i}, b_{j}$ 
non-isomorphic and I some non-empty index set. Given a regular initial conflict $\operatorname{stp}_{K}:\left\langle t p_{K}, \mathrm{ac}_{K}, \mathrm{ac}_{K}^{*}\right\rangle$, then $\mathcal{U}^{\mathcal{D}}\left(\operatorname{stp}_{K}\right)=\cup_{i \in I}\left\{t p_{C_{i}}: D_{1, i} \Leftarrow \rho_{1}, a_{i} \circ o_{1}\right.$

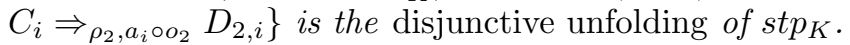

Remark 5 (disjunctive unfolding). The disjunctive unfolding of a regular conflict is non-empty, but might consist of less elements than literals in the disjunction $\vee_{i \in I} \exists\left(a_{i}: K \rightarrow C_{i}, c_{i}\right)$ : if a morphism $a_{i}$ does not satisfy the gluing condition of the derived spans, then also every extension morphism starting from there will not satisfy the gluing condition, and we can safely ignore that case from the disjunctive unfolding.

Theorem 5 (finite and complete unfolding). Consider an $\mathcal{M}$-adhesive system with initial transformation pairs for conflicts along plain rules. Given a rule pair $\left\langle\rho_{1}, \rho_{2}\right\rangle$ with set $\mathcal{S}$ of initial conflicts such that each initial conflict stp in $S$ is regular, then $\cup_{\text {stp } \in \mathcal{S}} \mathcal{U}^{\mathcal{D}}(s t p)$ is $\mathcal{M}$-complete w.r.t. parallel dependence. Moreover, $\cup_{s t p \in \mathcal{S}} \mathcal{U}^{\mathcal{D}}(s t p)$ is finite if $\mathcal{S}$ is finite.

It is possible to automatically check if some initial conflict is regular by using dedicated automated reasoning [22] as well as symbolic model generation for ACs [28] as follows. The reasoning mechanism [22] is shown to be refutationally complete ensuring that if the condition $\mathrm{ac}_{K} \wedge \mathrm{ac}_{K}^{*}$ of some initial conflict is unsatisfiable, this will be detected eventually. Moreover, the related symbolic model generation mechanism [28] is able to automatically transform each condition $\operatorname{ac}_{K} \wedge \mathrm{ac}_{K}^{*}$ into some disjunction $\vee_{i \in I} \exists\left(a_{i}: K \rightarrow C_{i}, c_{i}\right)$ with $c_{i}$ a negative remainder if such an equivalence holds.

\subsection{Unfolding for Rules with NACs}

We show that for rules with NACs initial conflicts are regular. This means that in this special case there exists a complete subset of conflicts that is finite (in the case of graphs and assuming finite rules). This conforms to the findings in $[17,20]$, where an $\mathcal{M}$-complete set of critical pairs - as specific subset of conflicts - for graph transformation rules with NACs was introduced [20] (and generalized to $\mathcal{M}$-adhesive transformation systems [17]).

Theorem 6 (regular initial conflicts for rules with NACs). Consider an $\mathcal{M}$-adhesive system with initial transformation pairs for conflicts along plain rules. Given some initial conflict $s t p_{K}:\left\langle t p_{K}, \mathrm{ac}_{K}, \mathrm{ac}_{K}^{*}\right\rangle$ for a pair of rules $\left\langle\rho_{1}, \rho_{2}\right\rangle$ with $\operatorname{ac}_{L_{i}}=\wedge_{j \in J} \neg \exists n_{j}: L_{i} \rightarrow N_{j}$ for $i=1,2$ and $J$ some finite index set, then it is regular. In particular, $\mathrm{ac}_{K} \wedge \mathrm{ac}_{K}^{*}$ is equivalent to a condition $\vee_{i \in I} \exists\left(a_{i}: K \rightarrow C_{i}, c_{i}\right)$ with $c_{i}=\wedge_{q \in Q} \neg \exists n_{q}$ a condition on $C_{i}$ and $I$ some non-empty index set.

The negative remainder $c_{i}$ of each literal in $\vee_{i \in I} \exists\left(a_{i}: K \rightarrow C_{i}, c_{i}\right)$ of a regular initial conflict for rules with NACs thus consists of a set of NACs. Intuitively this means that we obtain for each initial conflict an $\mathcal{M}$-complete subset of concrete conflicts by adding the context described by $a_{i}$. As long as no NAC from $c_{i}$ is violated we can extend such a concrete conflict to further ones. 
Corollary 1 (complete unfolding: rules with NACs). Consider an $\mathcal{M}$ adhesive system, with initial transformation pairs for conflicts along plain rules. Given a rule pair $\left\langle\rho_{1}, \rho_{2}\right\rangle$ with $\operatorname{ac}_{L_{i}}=\wedge_{j \in J} \neg \exists n_{j}: L_{i} \rightarrow N_{j}$ for $i=1,2$, then $\cup_{s t p \in \mathcal{S}} \mathcal{U}^{\mathcal{D}}(s t p)$ is $\mathcal{M}$-complete w.r.t. parallel dependence.

We show moreover that the initial conflict definition is a conservative extension of the critical pair definition for rules with NACs as given in $[17,20]$, i.e., we show that each conflict in the disjunctive unfolding of an initial conflict as chosen in the proof of Theorem 6 is a critical pair for rules with NACs.

Theorem 7 (conservative unfolding). In an $\mathcal{M}$-adhesive system with initial transformation pairs for conflicts along plain rules, if stp $p_{K}:\left\langle t p_{K}, \mathrm{ac}_{K}, \mathrm{ac}_{K}^{*}\right\rangle$ is an initial conflict for rules $\left\langle\rho_{1}, \rho_{2}\right\rangle$ with $\mathrm{ac}_{L_{i}}=\wedge_{j \in J} \neg \exists n_{j}: L_{i} \rightarrow N_{j}$ for $i=1,2$ and $J$ some finite index set, then each conflict as chosen in the proof of Theorem 6 in $\mathcal{U}^{\mathcal{D}}(s t p)$ is in particular a critical pair for $\left\langle\rho_{1}, \rho_{2}\right\rangle$ as given in [17, 20].

Example 4 (conservative unfolding). Consider again the rules from Example 1 (having only NACs as ACs) and their application to the graph $G=\bigcirc \bigcirc$. The corresponding transformation pair $t p_{G}$ is a critical pair for rules with NACs as given in $[17,20]$. This is because it is in particular a conflicting pair of transformations, and the morphism violating the NAC (since finding the three nodes) and therefore causing the conflict after applying the first rule to $G=\bigcirc \bigcirc$ obtaining some graph $H_{1}=\bigcirc \rightarrow \bigcirc \bigcirc$ is jointly surjective together with the corresponding co-match. As argued already in Example 2 this critical pair for rules with NACs belongs to the unfolding (and in particular to the disjunctive unfolding) of the unique AC initial conflict $s t p_{L_{1}+L_{2}}:\left\langle t p_{L_{1}+L_{2}}, \mathrm{ac}_{L_{1}+L_{2}}, \mathrm{ac}_{L_{1}+L_{2}}^{*}\right\rangle$.

\section{Conclusion and Outlook}

In this paper we have generalized the theory of initial conflicts (from plain rules, i.e. rules without application conditions) to rules with application conditions (ACs) in the framework of $\mathcal{M}$-adhesive transformation systems. We build on the notion of symbolic transformation pairs, since it turns out that it is not possible to find a complete subset of concrete conflicting transformation pairs in the case of rules with ACs. We have shown that initial conflicts are (minimally) complete w.r.t. parallel dependence as symbolic transformation pairs. Moreover, initial conflicts represent (analogous to the case of plain rules) proper subsets of critical pairs in the sense that for each critical pair (or also for each conflict), there exists a unique initial conflict representing it. We concluded the paper by showing sufficient conditions for finding unfoldings of initial conflicts that lead to (finite and) complete subsets of conflicts (as in the case of rules with NACs). Thereby we have shown that initial conflicts for rules with ACs represent a conservative extension of the critical pair theory for rules with NACs.

As future work we aim at finding further interesting classes allowing finite and (minimally) complete unfoldings into subsets of conflicts. This will serve as a guideline to be able to develop and implement efficient conflict detection techniques for rules with (specific) ACs, which has been an open challenge until today. 
We are moreover planning to develop (semi-)automated detection of unfoldings of initial conflicts of rules with arbitrary ACs using dedicated automated reasoning and model finding for graph conditions [22,26,28]. It would be interesting to investigate in which use cases initial conflicts (or critical pairs) are useful already as symbolic transformation pairs, and in which use cases we rather need to consider unfoldings indeed. This is in line with the research on multi-granular conflict detection $[3,18,24]$ investigating different levels of granularity that can be interesting from the point of view of applying conflict detection to different use cases. Finally, we plan to investigate conflict detection in the light of initial conflict theory for attributed graph transformation $[5,13,16]$, and in particular the case of rules with so-called attribute conditions more specifically. It would also be interesting to further investigate initial conflicts for transformation rules (with ACs) not following the DPO approach.

Acknowledgement. We thank Jens Kosiol for pointing out that the set of initial conflicts for plain rules is not only complete, but also minimally complete. We were able to transfer this result to rules with ACs in this paper. Many thanks also to the reviewers for their detailed and constructive comments helping to finalize the paper.

\section{References}

1. Azzi, G.G., Corradini, A., Ribeiro, L.: On the essence and initiality of conflicts in m-adhesive transformation systems. J. Log. Algebr. Methods Program. 109, $100482(2019)$

2. Bonchi, F., Gadducci, F., Kissinger, A., Sobociński, P., Zanasi, F.: Confluence of graph rewriting with interfaces. In: Yang, H. (ed.) ESOP 2017. LNCS, vol. 10201, pp. 141-169. Springer, Heidelberg (2017). https://doi.org/10.1007/978-3662-54434-1_6

3. Born, K., Lambers, L., Strüber, D., Taentzer, G.: Granularity of conflicts and dependencies in graph transformation systems. In: de Lara, J., Plump, D. (eds.) ICGT 2017. LNCS, vol. 10373, pp. 125-141. Springer, Cham (2017). https://doi. org/10.1007/978-3-319-61470-0_8

4. Ehrig, H., Ehrig, K., Habel, A., Pennemann, K.: Theory of constraints and application conditions: from graphs to high-level structures. Fundam. Inform. 74(1), 135-166 (2006)

5. Ehrig, H., Ehrig, K., Prange, U., Taentzer, G.: Fundamentals of Algebraic Graph Transformation. Monographs in Theoretical Computer Science. An EATCS Series. Springer, Heidelberg (2006). https://doi.org/10.1007/3-540-31188-2

6. Ehrig, H., Golas, U., Habel, A., Lambers, L., Orejas, F.: $\mathcal{M}$-adhesive transformation systems with nested application conditions. Part 2: embedding, critical pairs and local confluence. Fundam. Inform. 118(1-2), 35-63 (2012). https://doi.org/ 10.3233/FI-2012-705

7. Ehrig, H., Golas, U., Habel, A., Lambers, L., Orejas, F.: $\mathcal{M}$-adhesive transformation systems with nested application conditions. Part 1: parallelism, concurrency and amalgamation. Math. Struct. Comput. Sci. 24(4) (2014). https://doi.org/10. 1017/S0960129512000357

8. Ehrig, H., Habel, A.: Graph grammars with application conditions. In: Rozenberg, G., Salomaa, A. (eds.) The Book of L, pp. 87-100. Springer, Heidelberg (1986). https://doi.org/10.1007/978-3-642-95486-3_7 
9. Ehrig, H., Habel, A., Lambers, L., Orejas, F., Golas, U.: Local confluence for rules with nested application conditions. In: Ehrig, H., Rensink, A., Rozenberg, G., Schürr, A. (eds.) ICGT 2010. LNCS, vol. 6372, pp. 330-345. Springer, Heidelberg (2010). https://doi.org/10.1007/978-3-642-15928-2_22

10. Habel, A., Heckel, R., Taentzer, G.: Graph grammars with negative application conditions. Fundam. Inform. 26(3/4), 287-313 (1996). https://doi.org/10.3233/ FI-1996-263404

11. Habel, A., Pennemann, K.: Correctness of high-level transformation systems relative to nested conditions. Math. Struct. Comput. Sci. 19(2), 245-296 (2009). https://doi.org/10.1017/S0960129508007202

12. Hausmann, J.H., Heckel, R., Taentzer, G.: Detection of conflicting functional requirements in a use case-driven approach: a static analysis technique based on graph transformation. In: Tracz, W., Young, M., Magee, J. (eds.) Proceedings of the 24th International Conference on Software Engineering, ICSE 2002, pp. 105115. ACM (2002). https://doi.org/10.1145/581339.581355

13. Hristakiev, I., Plump, D.: Attributed graph transformation via rule schemata: Church-Rosser theorem. In: Milazzo, P., Varró, D., Wimmer, M. (eds.) STAF 2016. LNCS, vol. 9946, pp. 145-160. Springer, Cham (2016). https://doi.org/10.1007/ 978-3-319-50230-4_11

14. Knuth, D., Bendix, P.: Simple word problems in universal algebras. In: Leech, J. (ed.) Computational Problems in Abstract Algebra, pp. 263-297. Pergamon Press, Oxford (1970)

15. Koch, M., Mancini, L.V., Parisi-Presicce, F.: Graph-based specification of access control policies. J. Comput. Syst. Sci. 71(1), 1-33 (2005). https://doi.org/10.1016/ j.jcss.2004.11.002

16. Kulcsár, G., Deckwerth, F., Lochau, M., Varró, G., Schürr, A.: Improved conflict detection for graph transformation with attributes. In: Rensink, A., Zambon, E. (eds.) Proceedings GaM@ETAPS 2015. EPTCS, vol. 181, pp. 97-112 (2015). https://doi.org/10.4204/EPTCS.181.7

17. Lambers, L.: Certifying rule-based models using graph transformation. Ph.D. thesis, Berlin Institute of Technology (2009). http://opus.kobv.de/tuberlin/volltexte/ $2010 / 2522 /$

18. Lambers, L., Born, K., Kosiol, J., Strüber, D., Taentzer, G.: Granularity of conflicts and dependencies in graph transformation systems: a two-dimensional approach. J. Log. Algebr. Methods Program. 103, 105-129 (2019). https://doi.org/10.1016/ j.jlamp.2018.11.004

19. Lambers, L., Born, K., Orejas, F., Strüber, D., Taentzer, G.: Initial conflicts and dependencies: critical pairs revisited. In: Heckel, R., Taentzer, G. (eds.) Graph Transformation, Specifications, and Nets. LNCS, vol. 10800, pp. 105-123. Springer, Cham (2018). https://doi.org/10.1007/978-3-319-75396-6_6

20. Lambers, L., Ehrig, H., Orejas, F.: Conflict detection for graph transformation with negative application conditions. In: Corradini, A., Ehrig, H., Montanari, U., Ribeiro, L., Rozenberg, G. (eds.) ICGT 2006. LNCS, vol. 4178, pp. 61-76. Springer, Heidelberg (2006). https://doi.org/10.1007/11841883_6

21. Lambers, L., Ehrig, H., Orejas, F.: Efficient conflict detection in graph transformation systems by essential critical pairs. Electr. Notes Theor. Comput. Sci. 211, $17-26$ (2008)

22. Lambers, L., Orejas, F.: Tableau-based reasoning for graph properties. In: Giese, H., König, B. (eds.) ICGT 2014. LNCS, vol. 8571, pp. 17-32. Springer, Cham (2014). https://doi.org/10.1007/978-3-319-09108-2_2 
23. Lambers, L., Orejas, F.: Initial conflicts for transformation rules with nested application conditions (2020). arXiv:2005.05901 [cs.LO]

24. Lambers, L., Strüber, D., Taentzer, G., Born, K., Huebert, J.: Multi-granular conflict and dependency analysis in software engineering based on graph transformation. In: Chaudron, M., Crnkovic, I., Chechik, M., Harman, M. (eds.) Proceedings of the 40th International Conference on Software Engineering, ICSE 2018, pp. 716-727. ACM (2018). https://doi.org/10.1145/3180155.3180258

25. Ohlebusch, E.: Advanced Topics in Term Rewriting. Springer, Heidelberg (2002). https://doi.org/10.1007/978-1-4757-3661-8

26. Pennemann, K.: Development of correct graph transformation systems. Ph.D. thesis, University of Oldenburg, Germany (2009). http://oops.uni-oldenburg.de/ volltexte/2009/948/

27. Plump, D.: Hypergraph rewriting: Critical pairs and undecidability of confluence. In: Sleep, R., Plasmeijer, R., van Eekelen, M. (eds.) Term Graph Rewriting: Theory and Practice, pp. 201-213. Wiley, Chichester (1993)

28. Schneider, S., Lambers, L., Orejas, F.: Symbolic model generation for graph properties. In: Huisman, M., Rubin, J. (eds.) FASE 2017. LNCS, vol. 10202, pp. 226-243. Springer, Heidelberg (2017). https://doi.org/10.1007/978-3-662-54494-5_13 